Bull. Korean Math. Soc. 49 (2012), No. 5, pp. 1081-1088

http://dx.doi.org/10.4134/BKMS.2012.49.5.1081

\title{
A REMARK CONCERNING UNIVERSAL CURVATURE IDENTITIES ON 4-DIMENSIONAL RIEMANNIAN MANIFOLDS
}

\author{
Yunhee Euh, Chohee Jeong, and JeongHyeong Park
}

ABSTRACT. We shall prove the universality of the curvature identity for the 4-dimensional Riemannian manifold using a different method than that used by Gilkey, Park, and Sekigawa [5].

\section{Introduction}

Berger [1] derived a curvature identity on a 4-dimensional compact oriented Riemannian manifold $M=(M, g)$ from the generalized Gauss-Bonnet formula

$$
32 \pi^{2} \chi(M)=\int_{M} \tau^{2}-4|\rho|^{2}+|R|^{2} d v
$$

where $R$ is the curvature tensor, $\rho$ is the Ricci tensor and $\tau$ is the scalar curvature of $M$. The curvature identity is the quadratic equation which involves only the curvature tensor and not its covariant derivatives as follows:

$$
\frac{1}{4}\left(|R|^{2}-4|\rho|^{2}+\tau^{2}\right) g-\check{R}+2 \check{\rho}+L \rho-\tau \rho=0 .
$$

Here,

$$
\begin{gathered}
\check{R}: \check{R}_{i j}=\sum_{a, b, c} R_{a b c i} R^{a b c}, \quad \check{\rho}: \check{\rho}_{i j}=\sum_{a} \rho_{a i} \rho_{j}^{a}, \\
L:(L \rho)_{i j}=2 \sum_{a, b} R_{i a b j} \rho^{a b} .
\end{gathered}
$$

Euh, Park, and Sekigawa [2] proved that Equation (1) holds on the space of all Riemannian metrics on any 4-dimensional Riemannian manifold, and gave some applications of the curvature identity $[3,4]$. Labbi [7] showed the same

Received June 29, 2011; Revised August 23, 2011

2010 Mathematics Subject Classification. Primary 53B20, 53C20.

Key words and phrases. generalized Gauss-Bonnet formula, 4-dimensional curvature identity.

This work was supported by the National Research Foundation of Korea(NRF) grant funded by the Korea government(MEST) (2012-0005282) and by Korea Institute for Advanced Study(KIAS) grant funded by the Korea government(MEST).

(C)2012 The Korean Mathematical Society 
phenomena occurs for the higher dimensional cases by using purely algebraic computations in the ring of double forms and also provided some applications of the curvature identity in [8]. Recently, Gilkey, Park, and Sekigawa [5] gave a new proof of the curvature identity using heat trace methods. Here, we raise the following question:

Question. Is there another curvature identity such as the quadratic curvature identity (1) which holds on any 4-dimensional Riemannian manifold $(M, g)$ ?

In the present paper, we shall give an answer to the above Question with a different method given by [5]. Namely, we shall prove the following theorem.

Main Theorem. The curvature identity (1) is universal as a symmetric 2form valued quadratic curvature identity for a 4-dimensional Riemannian manifold.

The authors would like to express their thanks to Professor K. Sekigawa and E. Puffini for their helpful comments and valuable suggestions. The authors are also very grateful to the anonymous referee for the useful comments which improved the manuscript.

\section{Preliminary}

Let $M$ be an $m$-dimensional Riemannian manifold and $\mathcal{I}_{m, n}^{2}(n$ is even) be the space of symmetric 2-form valued invariants which are homogeneous of degree $n$ in the derivatives of the metric on $M$. In [5], Gilkey, Park, and Sekigawa proved that the universality of the curvature identity in the setting of the space $\mathcal{I}_{4,4}^{2}$. Now, we set

$$
\begin{gathered}
\Phi_{1}:=|R|^{2} g, \quad \Phi_{2}:=|\rho|^{2} g, \quad \Phi_{3}:=\tau^{2} g, \quad \Phi_{4}:=\check{R}, \quad \Phi_{5}:=\check{\rho}, \\
\Phi_{6}:=L \rho, \quad \Phi_{7}:=\tau \rho, \quad \Phi_{8}=(\triangle \tau) g, \quad \Phi_{9}=\operatorname{Hess} \tau, \quad \Phi_{10}=\tilde{\triangle} \rho,
\end{gathered}
$$

where $\tilde{\triangle} \rho$ denotes the rough Laplacian acting on the Ricci tensor $\rho$, namely locally expressed by $(\tilde{\triangle} \rho)_{i j}=\sum_{a} \nabla^{a} \nabla_{a} \rho_{i j}$. Then, we have the following:

Lemma 2.1 ([5]).

(1) $\mathcal{I}_{m, 0}^{2}=\operatorname{Span}\{g\}$.

(2) $\mathcal{I}_{m, 2}^{2}=\operatorname{Span}\{\tau g, \rho\}$.

(3) $\mathcal{I}_{m, 4}^{2}=\operatorname{Span}\left\{\Phi_{1}, \Phi_{2}, \ldots, \Phi_{7}, \Phi_{8}, \Phi_{9}, \Phi_{10}\right\}$.

In $[5,6]$, Gilkey et al. proved that the curvature identity

$$
\frac{\lambda}{4} \Phi_{1}-\lambda \Phi_{2}+\frac{\lambda}{4} \Phi_{3}-\lambda \Phi_{4}+2 \lambda \Phi_{5}+\lambda \Phi_{6}-\lambda \Phi_{7}=0
$$

for any constant $\lambda(\neq 0)$, is the only universal curvature identity of this form if $m=4$ ([5], Theorem 1.2(3) and Lemma 1.4(2)). We may easily check that the curvature identities (1) and (2) are equivalent to each other. We emphasize 
that the invariance theory established by H. Weyl plays an important role in their proof of [5, Theorem 1.2].

Here, we give another direct proof for the same result by using several test Riemannian manifolds of dimension 4.

\section{Proof of Main Theorem}

We assume that the equality

$$
\sum_{i=1}^{10} c_{i} \Phi_{i}=0
$$

holds for all 4-dimensional Riemannian manifolds. To prove Main Theorem, it is sufficient to prove that $c_{1}=\frac{\lambda}{4}, c_{2}=-\lambda, c_{3}=\frac{\lambda}{4}, c_{4}=-\lambda, c_{5}=2 \lambda, c_{6}=\lambda$, $c_{7}=-\lambda, c_{8}=c_{9}=c_{10}=0$.

Applying (3) to the test manifolds in Cases I, II, III, IV and V, we will determine the coefficients $c_{i}$ 's such that $\sum_{i} c_{i} \Phi_{i}=0(i=1, \ldots, 10)$ by applying the method of universal examples. This is the way we can show whether the curvature identity (1) is universal or not.

Case I. Let $M$ be a locally product of Riemannian surfaces $M^{2}(a)$ and $M^{2}(b)$ of nonzero constant Gaussian curvatures $a$ and $b$. Let $\left\{e_{1}, e_{2}\right\}$ and $\left\{e_{3}, e_{4}\right\}$ be the orthonormal basis of $M^{2}(a)$ and $M^{2}(b)$, respectively. Then we have the following:

(4)

$$
\begin{gathered}
\Phi_{1}=4\left(a^{2}+b^{2}\right) I, \quad \Phi_{2}=2\left(a^{2}+b^{2}\right) I, \quad \Phi_{3}=4(a+b)^{2} I, \\
\Phi_{4}=\left(\begin{array}{cccc}
2 a^{2} & 0 & 0 & 0 \\
0 & 2 a^{2} & 0 & 0 \\
0 & 0 & 2 b^{2} & 0 \\
0 & 0 & 0 & 2 b^{2}
\end{array}\right), \quad \Phi_{5}=\left(\begin{array}{cccc}
a^{2} & 0 & 0 & 0 \\
0 & a^{2} & 0 & 0 \\
0 & 0 & b^{2} & 0 \\
0 & 0 & 0 & b^{2}
\end{array}\right), \\
\Phi_{6}=\left(\begin{array}{cccc}
2 a^{2} & 0 & 0 & 0 \\
0 & 2 a^{2} & 0 & 0 \\
0 & 0 & 2 b^{2} & 0 \\
0 & 0 & 0 & 2 b^{2}
\end{array}\right), \quad \Phi_{7}=2(a+b)\left(\begin{array}{cccc}
a & 0 & 0 & 0 \\
0 & a & 0 & 0 \\
0 & 0 & b & 0 \\
0 & 0 & 0 & b
\end{array}\right),
\end{gathered}
$$

From (4), we can get two different equations such that $\sum_{i} c_{i} \Phi_{i}=0$ :

(I-i) (1,1)-component (or $(2,2)$-component)

$\left(4 c_{1}+2 c_{2}+4 c_{3}+2 c_{4}+c_{5}+2 c_{6}+2 c_{7}\right) a^{2}+\left(8 c_{3}+2 c_{7}\right) a b+\left(4 c_{1}+2 c_{2}+4 c_{3}\right) b^{2}=0$.

(I-ii) (3,3)-component (or $(4,4)$-component)

$\left(4 c_{1}+2 c_{2}+4 c_{3}\right) a^{2}+\left(8 c_{3}+2 c_{7}\right) a b+\left(4 c_{1}+2 c_{2}+4 c_{3}+2 c_{4}+c_{5}+2 c_{6}+2 c_{7}\right) b^{2}=0$. 
We set $c_{7}=-\lambda$. Then from (I-i) and (I-ii), we have the following relations:

$$
\begin{aligned}
& c_{3}=\frac{1}{4} \lambda, \\
& 4 c_{1}+2 c_{2}=-\lambda, \\
& 2 c_{4}+c_{5}+2 c_{6}=2 \lambda .
\end{aligned}
$$

Case II. Let $M$ be a product of 3-dimensional Riemannian manifold $M^{3}(a)$ of nonzero constant sectional curvature $a$ and a real line $\mathbb{R}$. Let $\left\{e_{1}, e_{2}, e_{3}\right\}$ be the orthonormal basis of $M^{3}(a)$. Then we have the following:

(6)

$$
\begin{gathered}
\Phi_{1}=12 a^{2} I, \quad \Phi_{2}=12 a^{2} I, \quad \Phi_{3}=36 a^{2} I \\
\Phi_{4}=4 a^{2}\left(\begin{array}{cccc}
1 & 0 & 0 & 0 \\
0 & 1 & 0 & 0 \\
0 & 0 & 1 & 0 \\
0 & 0 & 0 & 0
\end{array}\right), \quad \Phi_{5}=4 a^{2}\left(\begin{array}{cccc}
1 & 0 & 0 & 0 \\
0 & 1 & 0 & 0 \\
0 & 0 & 1 & 0 \\
0 & 0 & 0 & 0
\end{array}\right), \\
\Phi_{6}=8 a^{2}\left(\begin{array}{llll}
1 & 0 & 0 & 0 \\
0 & 1 & 0 & 0 \\
0 & 0 & 1 & 0 \\
0 & 0 & 0 & 0
\end{array}\right), \quad \Phi_{7}=12 a^{2}\left(\begin{array}{cccc}
1 & 0 & 0 & 0 \\
0 & 1 & 0 & 0 \\
0 & 0 & 1 & 0 \\
0 & 0 & 0 & 0
\end{array}\right),
\end{gathered}
$$

From (6), we can get two different equations such that $\sum_{i} c_{i} \Phi_{i}=0$ : (II-i) (1,1)-component $((2,2)$ or $(3,3)$-component)

$$
\left(3 c_{1}+3 c_{2}+9 c_{3}+c_{4}+c_{5}+2 c_{6}+3 c_{7}\right) a^{2}=0 .
$$

(II-ii) (4,4)-component

$$
\left(c_{1}+c_{2}+3 c_{3}\right) a^{2}=0 .
$$

From (II-i) and (II-ii), we have the following relation:

$$
c_{4}+c_{5}+2 c_{6}+3 c_{7}=0,
$$

and hence, since $c_{7}=-\lambda$, we get

$$
c_{4}+c_{5}+2 c_{6}=3 \lambda .
$$

From (5) and (7), we have

$$
c_{4}=-\lambda, \quad c_{5}+2 c_{6}=4 \lambda .
$$

Case III. Let $M=M^{4}(a)$ be a space form of nonzero constant sectional curvature $a$. Then we have the following:

$$
\begin{gathered}
\Phi_{1}=24 a^{2} I, \quad \Phi_{2}=36 a^{2} I, \quad \Phi_{3}=144 a^{2} I, \\
\Phi_{4}=6 a^{2} I, \quad \Phi_{5}=9 a^{2} I, \quad \Phi_{6}=18 a^{2} I, \\
\Phi_{7}=36 a^{2} I, \quad \Phi_{8}=\Phi_{9}=\Phi_{10}=0 .
\end{gathered}
$$


From (9), we can get an equation such that $\sum_{i} c_{i} \Phi_{i}=0$ :

(III) (1,1)-component $((2,2),(3,3)$, or $(4,4)$-component)

$$
\left(24 c_{1}+36 c_{2}+144 c_{3}+6 c_{4}+9 c_{5}+18 c_{6}+36 c_{7}\right) a^{2}=0 .
$$

From (III-i), we have the following relation:

$$
8 c_{1}+12 c_{2}+48 c_{3}+2 c_{4}+3 c_{5}+6 c_{6}+12 c_{7}=0 .
$$

Since $c_{7}=-\lambda$, from (5) and (8), we get

$$
c_{1}=\frac{\lambda}{4}, \quad c_{2}=-\lambda .
$$

Case IV. ([3], Example 3.7) Let $\mathfrak{g}=\operatorname{span}_{\mathbb{R}}\left\{e_{1}, e_{2}, e_{3}, e_{4}\right\}$ be a 4-dimensional real Lie algebra equipped with the following Lie bracket operation:

$$
\begin{aligned}
& {\left[e_{1}, e_{2}\right]=a e_{2}, \quad\left[e_{1}, e_{3}\right]=-a e_{3}-b e_{4}, \quad\left[e_{1}, e_{4}\right]=b e_{3}-a e_{4},} \\
& {\left[e_{2}, e_{3}\right]=0, \quad\left[e_{2}, e_{4}\right]=0, \quad\left[e_{3}, e_{4}\right]=0,}
\end{aligned}
$$

where $a(\neq 0), b$ are constant. We define an inner product $\langle$,$\rangle on \mathfrak{g}$ by $\left\langle e_{i}, e_{j}\right\rangle=$ $\delta_{i j}$. Let $G$ be a connected and simply connected solvable Lie group with the Lie algebra $\mathfrak{g}$ of $G$ and $g$ the $G$-invariant Riemannian metric on $G$ determined by $\langle$,$\rangle . From (11), by direct calculations, we have$

$$
\begin{aligned}
& R_{1212}=a^{2}, \quad R_{1313}=a^{2}, \quad R_{1414}=a^{2}, \\
& R_{2323}=-a^{2}, \quad R_{2424}=-a^{2}, \quad R_{3434}=a^{2},
\end{aligned}
$$

and otherwise being zero up to sign.

$$
(\rho)=\left(\begin{array}{cccc}
-3 a^{2} & 0 & 0 & 0 \\
0 & a^{2} & 0 & 0 \\
0 & 0 & -a^{2} & 0 \\
0 & 0 & 0 & -a^{2}
\end{array}\right), \quad \tau=-4 a^{2} .
$$

Then, we have the following:

$$
\begin{gathered}
\Phi_{1}=24 a^{4} I, \quad \Phi_{2}=12 a^{4} I, \quad \Phi_{3}=16 a^{4} I, \quad \Phi_{4}=6 a^{4} I, \\
\Phi_{5}=a^{4}\left(\begin{array}{cccc}
9 & 0 & 0 & 0 \\
0 & 1 & 0 & 0 \\
0 & 0 & 1 & 0 \\
0 & 0 & 0 & 1
\end{array}\right), \quad \Phi_{6}=2 a^{4}\left(\begin{array}{cccc}
1 & 0 & 0 & 0 \\
0 & 1 & 0 & 0 \\
0 & 0 & 5 & 0 \\
0 & 0 & 0 & 5
\end{array}\right), \\
\Phi_{7}=4 a^{4}\left(\begin{array}{cccc}
3 & 0 & 0 & 0 \\
0 & -1 & 0 & 0 \\
0 & 0 & 1 & 0 \\
0 & 0 & 0 & 1
\end{array}\right), \quad \Phi_{10}=a^{4}\left(\begin{array}{cccc}
8 & 0 & 0 & 0 \\
0 & -8 & 0 & 0 \\
0 & 0 & -4 & 0 \\
0 & 0 & 0 & -4
\end{array}\right),
\end{gathered}
$$

From (13), we can get three different equations such that $\sum_{i} c_{i} \Phi_{i}=0$ : (IV-i) (1,1)-component

$$
\left(24 c_{1}+12 c_{2}+16 c_{3}+6 c_{4}+9 c_{5}+2 c_{6}+12 c_{7}+8 c_{10}\right) a^{4}=0 .
$$


(IV-ii) (2,2)-component

$$
\left(24 c_{1}+12 c_{2}+16 c_{3}+6 c_{4}+c_{5}+2 c_{6}-4 c_{7}-8 c_{10}\right) a^{4}=0 .
$$

(IV-iii) (3,3)-component (or (4,4)-component)

$$
\left(24 c_{1}+12 c_{2}+16 c_{3}+6 c_{4}+c_{5}+10 c_{6}+4 c_{7}-4 c_{10}\right) a^{4}=0 .
$$

Thus, from (14), taking account of (5), (8), (10) and $a \neq 0$, we have

$$
-20 \lambda+9 c_{5}+2 c_{6}+8 c_{10}=0 .
$$

Thus, from (15), we have

$$
-4 \lambda+c_{5}+2 c_{6}+-8 c_{10}=0 .
$$

Then, from (17) and (18), we have

$$
5 c_{5}+2 c_{6}=12 \lambda .
$$

Thus, from (8) and (19), we have

$$
c_{5}=2 \lambda, \quad c_{6}=\lambda .
$$

Thus, (17) and (20), we have

$$
c_{10}=0 .
$$

Case V. Let $M$ be the Riemannian product of Riemannian surfaces $\left(M_{1}, g_{1}\right)$ and $\left(M_{2}, g_{2}\right)$, where the Riemannian metrics $g_{1}$ and $g_{2}$ are given locally by

$$
\left(g_{1}\right)=\left(\begin{array}{cc}
e^{2 \sigma_{1}} & 0 \\
0 & e^{2 \sigma_{1}}
\end{array}\right), \quad \sigma_{1}=x_{1}^{2}+x_{2}^{2}
$$

and

$$
\left(g_{2}\right)=\left(\begin{array}{cc}
e^{2 \sigma_{2}} & 0 \\
0 & e^{2 \sigma_{2}}
\end{array}\right), \quad \sigma_{2}=x_{3}^{2}+x_{4}^{2}
$$

We set

$$
e_{1}=\frac{1}{e^{\sigma_{1}}} \frac{\partial}{\partial x_{1}}, \quad e_{2}=\frac{1}{e^{\sigma_{1}}} \frac{\partial}{\partial x_{2}}, \quad e_{3}=\frac{1}{e^{\sigma_{2}}} \frac{\partial}{\partial x_{3}}, \quad e_{4}=\frac{1}{e^{\sigma_{2}}} \frac{\partial}{\partial x_{4}} .
$$

We denote by $K_{1}$ and $K_{2}$ the Gaussian curvatures of $\left(M_{1}, g_{1}\right)$ and $\left(M_{2}, g_{2}\right)$, respectively. Then we have

$$
K_{1}=-4 e^{-2 \sigma_{1}}, \quad K_{2}=-4 e^{-2 \sigma_{2}} .
$$

Thus, from (22), we have the scalar curvature

$$
\tau=-8 e^{-2 \sigma_{1}}-8 e^{-2 \sigma_{2}} .
$$

Finally, we have

$$
\Phi_{8}=-64\left(e^{-4 \sigma_{1}}\left(2 \sigma_{1}-1\right)+e^{-4 \sigma_{2}}\left(2 \sigma_{2}-1\right)\right) I, \quad \Phi_{9}=\left(\begin{array}{cc}
A & 0 \\
0 & B
\end{array}\right),
$$


where

$$
\begin{gathered}
A=-32 e^{-4 \sigma_{1}}\left(\begin{array}{cc}
6 x_{1}^{2}-2 x_{2}^{2}-1 & 8 x_{1} x_{2} \\
8 x_{1} x_{2} & -2 x_{1}^{2}+6 x_{2}^{2}-1
\end{array}\right), \\
B=-32 e^{-4 \sigma_{1}}\left(\begin{array}{cc}
6 x_{3}^{2}-2 x_{4}^{2}-1 & 8 x_{3} x_{4} \\
8 x_{3} x_{4} & -2 x_{3}^{2}+6 x_{4}^{2}-1
\end{array}\right) .
\end{gathered}
$$

Then, from (3) and (23), since the curvature identity (1) holds for any 4dimensional manifold, taking account of (5), (8), (10), (20) and (21), we have the following coefficients $c_{i}$ 's:

$$
\begin{aligned}
& c_{1}=\frac{\lambda}{4}, \quad c_{2}=-\lambda, \quad c_{3}=\frac{\lambda}{4}, \quad c_{4}=-\lambda, \quad c_{5}=2 \lambda, \\
& c_{6}=\lambda, \quad c_{7}=-\lambda, \quad c_{8}=0, \quad c_{9}=0, \quad c_{10}=0 .
\end{aligned}
$$

From the above observation, we see that Equation (1) is unique on a 4dimensional Riemannian manifold. That is, the curvature identity (1) for a 4-dimensional Riemannian manifold is universal.

Remark 3.1. The universal relation still holds in the pseudo-Riemannian setting from the appropriate adjustments of sign of the metric in the test manifold. We refer to [9].

\section{References}

[1] M. Berger, Quelques formules de variation pour une structure riemannienne, Ann. Sci. École Norm. Sup. 43 (1970), 285-294.

[2] Y. Euh, J. H. Park, and K. Sekigawa, A Curvature identity on a 4-dimensional Riemannian manifold, Results. Math., in press, doi 10.1007/s00025-011-0164-3.

[3] _ A generalization of a 4-dimensional Einstein manifold, to appear Mathematica Slovaca.

[4] Critical metrics for squared-norm functionals of the curvature on 4-dimensional manifolds, Differential Geom. Appl. 29 (2011), no. 5, 642-646.

[5] P. Gilkey, J. H. Park, and K. Sekigawa, Universal curvature identities, Differential Geom. Appl. 29 (2011), no. 6, 770-778.

[6] _ The spanning set, unpublished.

[7] M.-L. Labbi, Variational properties of the Gauss-Bonnet curvatures, Calc. Var. Partial Differential Equations 32 (2008), no. 2, 175-189.

[8] _ On generalized Einstein metrics, Balkan J. Geom. Appl. 15 (2010), no. 2, 69-77.

[9] E. Puffini, Curvature identities, unpublished.

[10] H. Weyl, Reine Infinitesimalgeometrie, Math. Z. 2 (1918), no. 3-4, 384-411.

YunHEE EuH

Department of Mathematics

SUNGKYUNKWAN UNIVERSITY

SuwON 440-746, KorEA

E-mail address: prettyfish@skku.edu

Chohee Jeong

Department of Mathematics

SUNGKYUNKWAN UNIVERSITY

Suwon 440-746, Korea

E-mail address: chohee1108@skku.edu 
JeOngHyeong PARK

Department of Mathematics

SUNGKYUNKWAN UNIVERSITY

SuWON 440-746, KoreA

E-mail address: parkj@skku.edu 